\title{
Polymorphic MicroRNA-Target Interactions: A Novel Source of Phenotypic Variation
}

\author{
M. Georges, * A. Clop, * F. MarcQ, ${ }^{*}$ H. Takeda, ${ }^{*}$ D. Pirottin, $*$ S. Hiard, ${ }^{*}$ X. Tordoir, $*$ \\ F. Caiment, ${ }^{*}$ F. Meish, ${ }^{*}$ B. Bibé, ${ }^{\dagger}$ J. BouiX,${ }^{\dagger}$ J.M. Elsen,${ }^{\dagger}$ F. Eychenne ${ }^{\dagger}$ E. Laville, ${ }^{q \dagger}{ }^{\dagger}$ \\ C. Larzul ${ }^{\dagger}$ D. Milenkovic ${ }^{\S}$ J. Tobin, ${ }^{\dagger}$ AND C. Charlier* ${ }^{*}$ \\ *Unit of Animal Genomics, Department of Animal Production, Faculty of Veterinary Medicine \& CBIG, \\ University of Liège (B43), 4000-Liège, Belgium; ${ }^{*}$ Cardiovascular and Metabolic Diseases, Wyeth Research, \\ Cambridge, Massachusetts 02140; INRA-SAGA, Institut National de la Recherche Agronomique-Station \\ d'Amélioration Génétique des Animaux, BP 52627-31326 Castanet-Tolosan CEDEX, France; \\ "I Station de Recherches sur la Viande, INRA, Theix, 63122 Saint-gene`s-Champanelle, France;

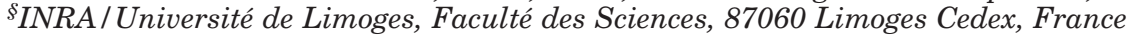

\begin{abstract}
Studying the muscular hypertrophy of Texel sheep by forward genetics, we have identified an A-to-G transition in the 3'UTR of the $G D F 8$ gene that reveals an illegitimate target site for microRNAs $m i R-1$ and $m i R-206$ that are highly expressed in skeletal muscle. This causes the down-regulation of this muscle-specific chalone and hence contributes to the muscular hypertrophy of Texel sheep. We demonstrate that polymorphisms which alter the content of putative miRNA target sites are common in human and mice, and provide evidence that both conserved and nonconserved target sites are selectively constrained. We speculate that these polymorphisms might be important mediators of phenotypic variation including disease. To facilitate studies along those lines, we have constructed a database (www.patrocles.org) listing putative polymorphic microRNA-target interactions.
\end{abstract}

Animal breeders have been conducting an unprecedented phenotype-driven screen for thousands of years, targeting behavioral, morphological, physiological, and pathological traits. This has resulted in the creation of numerous breeds that exhibit often spectacular phenotypic differences. Using the recently developed tools for genome analysis, animal geneticists are now attempting to identify the mutations that underlie this remarkable phenotypic plasticity. By doing so, they aim at contributing to a better molecular understanding of the genotype-phenotype relationship and adaptation, as well as at developing knowledge and tools allowing more proficient selection schemes (see, e.g., Andersson and Georges 2004).

One of the phenotypes that - for obvious reasons - has received considerable attention in livestock species is muscular development. Hypermuscled breeds, including Belgian Blue cattle, Piétrain pigs, and Callipyge sheep, have served as starting material for positional cloning efforts which led to the identification of structural mutations in myostatin (GDF8) (see, e.g., Grobet et al. 1997) and the calcium release channel (CRC) (Fujii et al. 1991), as well as regulatory mutation controlling the expression levels of insulin-like growth factor 2 (IGF2) (Van Laere et al. 2003) and delta-like 1 (DLK1) (Charlier et al. 2001; Freking et al. 2002; Davis et al. 2004).

We herein describe the recent positional identification of a mutation in the GDF8 $3^{\prime}$ UTR that creates an illegitimate target site for two miRNA that are highly expressed in skeletal muscle. The ensuing down-regulation of GDF8 contributes to the muscular hypertrophy of Texel sheep (Clop et al. 2006). We pursue by demonstrating that large numbers of single-nucleotide polymorphisms (SNPs), particularly in human and mice, have the potential to affect the interaction between miRNAs and their targets and may thereby be important mediators of phenotypic variation. The corresponding information is compiled in the open access Patrocles database (Clop et al. 2006; S. Hiard et al., in prep.).

\section{A MUTATION CREATING AN ILLEGITIMATE MIRNA TARGET SITE IN THE GDF8 3'UTR IS A QUANTITATIVE TRAIT NUCLEOTIDE AFFECTING MUSCULARITY IN SHEEP}

\section{A QTL with Major Effect on Muscularity Maps to Sheep Chromosome 2}

Texel sheep are famous and hence utilized worldwide for their pronounced muscular hypertrophy. This is especially true for the hypermuscled strain of Belgian Texel. To gain a better understanding of the molecular basis of this economically important phenotype, we generated a Romanov $\times$ Belgian Texel $\mathrm{F}_{2}$ cross counting 278 individuals. Romanov sheep are characterized by a mediocre muscular development but are very fertile. $\mathrm{F}_{2}$ animals were slaughtered at a target weight of $39 \mathrm{~kg}$ for the males and 35 $\mathrm{kg}$ for the females, and 56 phenotypes quantifying carcass composition were measured on each individual. The raw phenotypes were precorrected for a number of fixed effects and covariates including sex, year, slaughter plant, and final weight. All $F_{0}, F_{1}$, and $F_{2}$ animals were genotyped for 160 microsatellite markers spanning the sheep genome. Marker maps were constructed and shown to be in good agreement with published maps. The genome was scanned for quantitative trait loci (QTL) using linear regression (Haley et al. 1994), significance thresholds determined by 
phenotype permutation (Doerge and Churchill 1996), and confidence intervals (CI) determined by bootstrapping (Visscher et al. 1996). A highly significant effect on virtually all phenotypes measuring muscularity or fat deposition was found in the pericentromeric region of chromosome 2 (OAR2). This QTL typically explained about $25 \%$ of the breed difference, about $20 \%$ of the $\mathrm{F}_{2}$ variance, and acted mostly in a partially recessive way.

The CI for the QTL location spanned $10 \mathrm{cM}$ including the OAR2 centromere. Notably, the orthologous marker interval is known to harbor GDF8 in the bovine. Loss-offunction mutations in the $G D F 8$ gene are known to have dramatic effects on muscle mass in mouse, bovine, and human (hence the alternative name myostatin), making this gene the perfect positional candidate (see, e.g., Lee 2004). We thus sequenced the coding portions from the gene after PCR amplification of the exons from genomic DNA of Texel and Romanov but did not find a single sequence difference. The same experiment was repeated using cDNA from skeletal muscle of Texel and Romanov, yielding equivalent amounts of product with identical size, sequence, and thus, coding potential. Moreover, northern blot analysis revealed a band of the expected size and with very comparable intensity in Texel and Romanov. At first glance, these results did not support a role for $G D F 8$ in the muscular hypertrophy of Texel sheep.

\section{Fine-mapping Positions the QTL in the Immediate Vicinity of the $G D F 8$ Gene}

To refine the map position of the QTL, we first increased the marker density in the CI. We then applied two fine-mapping strategies. In a first instance, we identified $\mathrm{F}_{2}$ ram having inherited an intact Texel chromosome from one parent, and a recombinant Texel-Romanov chromosome recombining in the CI from the other. The recombinant chromosome was of Texel descent for most of the CI ( $~ 3 / 4$ on the OAR2p side), and of Romanov descent for the remaining quarter of the $\mathrm{CI}$ (on the OAR2q side). We generated 43 offspring from this ram, reared them until 35-39 kg, slaughtered them, and measured the same set of phenotypes as before. Sorting the offspring according to the paternal OAR2 homolog clearly indicated that the ram was heterozygous $Q q$ for the QTL $(p=0.0013)$, thus positioning the QTL in the $1 / 4$ portion of the CI for which the ram was heterozygous Texel/Romanov.

In a second instance, we genotyped 42 hypermuscled Belgian Texels and 108 animals from 16 other breeds for all markers available in the CI. We reasoned that selection for meatiness in Belgian Texel might have caused the near fixation of a mutation increasing muscularity, hence causing a local reduction in genetic variation ("selective sweep"). A highly significant reduction in genetic variation was observed for most of the CI. However, the signal maximized on the OAR $2 q$ side of the interval, in agreement with the results obtained with the recombinant $F_{2}$ ram. More specifically, the signature of selection was highest for marker BULGE20, for which the homozygosity was $89 \%$ in Texels versus $15 \%$ in controls. BULGE20 is located less than one megabase from $G D F 8$, strongly encouraging us to examine the myostatin gene more closely.

\section{A $G$ to $A$ Substitution in the $G D F 8$ 3'UTR Is a Strong Genetic QTN Candidate}

We resequenced $10.5 \mathrm{~kb}$ spanning the entire $G D F 8$ gene including $3.5 \mathrm{~kb}$ of upstream, and $1.9 \mathrm{~kb}$ of downstream, sequence for three Texel and seven control animals. This identified 20 SNPs. As expected based on the previous experiments, none of these would affect the predicted GDF8 open reading frame. Six SNPs were located upstream of the transcription start site, two in the $5^{\prime} \mathrm{UTR}$, eight in intron 1, three in intron 2, and one in the $3^{\prime}$ UTR. However, none of them fell in a highly conserved gene segment suggestive of functionally important regulatory elements.

All 20 SNPs were genotyped on 42 hypermuscled Belgian Texels, 90 control animals (representing 11 breeds), and four rams known with virtual certainty to be heterozygous $Q q$ for the QTL. The latter corresponded to the previously described "recombinant" $F_{2}$ ram, as well as to the three $F_{1}$ rams utilized to generate the Romanov $x$ Texel $F_{2}$ population. Indeed, sorting the $F_{2}$ offspring based on the homolog inherited from the sire in the CI revealed a consistent allele substitution effect in the three $\mathrm{F}_{1}$ sire families.

The first striking observation was the virtual monomorphism of the GDF8 gene in Texels contrasting with the considerable polymorphism of the same gene among controls. This observation was consistent with the occurrence-in Texel — of a selective sweep driven by an advantageous $G D F 8$ mutation. Moreover, by virtue of the homozygosity of at least one of the four $Q q$ rams, all but two ( $g-2449 C$ $G$ and $g+6723 G-A)$ of the 20 SNPs could be excluded as being causal. More recently, the same approach applied to sheep populations in New Zealand led to the exclusion of the $g-2449 C-G$ SNP as well (J. McEwan, pers. comm.), leaving $g+6723 G-A$ as the only putative causal mutation. Finally, and consistent with the previous findings, the $g+6723 G-A$ SNP is the only one for which one allele $(A)$ is virtually Texel-specific (allelic frequency $99 \%$ in Texel versus $1 \%$ in controls). Altogether, these observations strongly incriminated $G D F 8$ and the $g+6723 G-A$ SNP in its 3'UTR as causing the observed QTL effect.

\section{miRNA-mediated Translation Inhibition of Mutant GDF8 Transcripts Underlie the QTL Effect}

Careful examination of the sequence context of $g+6723 G-A$ indicated that the $A$ allele reveals one of the 3'UTR octamer motifs (ACATTCCA) discovered by Xie et al. (2005) on the basis of their unusually high motif conservation score (MCS) and thought to correspond to miRNA target sites. We simulated all the possible nucleotide substitutions in the 3'UTR of the ovine GDF8 gene (accounting for a transition rate twice as large as the transversion rate) and demonstrated that only $4.5 \%$ of these would create one of the 540 motifs described by Xie et al. (2005), whereas only $0.88 \%$ would create an octamer with an equally high MCS. This indicated that our observation was not trivial, and suggested that the $g+6726 \mathrm{~A}$ allele might create an illegitimate miRNA target site resulting in posttranscriptional GDF8 down-regulation and hence in an increase in muscle mass. 
ACATTCCA is showing perfect Watson-Crick basepairing with nucleotides $1-8$ of $m i R-1$ and $m i R-206$, and near-perfect base-pairing (one G:U pair) with $m i R-122$, suggesting that one or several of these miRNAs might be mediating the hypothesized effect. Using primers targeting conserved flanking sequences, we amplified and sequenced the corresponding pri-miRNA genes from sheep genomic DNA demonstrating their conservation. Using primer extension, we then demonstrated that $m i R$ 1 and $m i R-206$ (but not $m i R-122 a$ ) were indeed preferentially and highly expressed in skeletal muscle. These findings are in agreement with more recent data in human and mice (see, e.g., Farh et al. 2005) and lent further support to our hypothesis.

Our model makes at least two in vivo testable predictions: (1) GDF8 protein levels should be reduced in Texel sheep when compared to controls as a result of translational inhibition of the mutant mRNA, and (2) mutant $G D F 8$ mRNA levels might be reduced when compared to wild-type $G D F 8$ mRNA levels as a result of accelerated degradation of mutant mRNA (see, e.g., Lim et al. 2005). To test the first prediction, we measured the levels of circulating GDF8 by immunoprecipitation followed by western blotting, a proven quantitative procedure that was previously successfully utilized in primates and rodents (see, e.g., Zimmers et al. 2002; Schuelke et al. 2004). A consistent threefold reduction in GDF8 levels was indeed observed in the serum of Texel sheep when compared to wild-type animals. To test the second prediction, we measured GDF8 mRNA allelic imbalance in skeletal muscle of heterozygous $A G$ individuals. Wild-type and mutant mRNA were amplified by hot-stop RT-PCR, distinguished by virtue of the $H p y C H 4 I V$ restriction site that is present in the wild-type but not mutant allele, and quantified after denaturing acrylamide gel electrophoresis using a phosphorimager. The ratios of mutant versus wild-type intensities were compared with those obtained from $A G$ genomic DNA as well as with a calibration curve, indicating a 1.5-fold reduction of mutant versus wild-type mRNA. Both in vivo predictions were thus met, in agreement with our model.

To provide direct evidence that the $g+6723 G-A$ SNP indeed affects the interaction between the GDF8 3'UTR and the miRNAs $m i R-1$ and $m i R-206$, we generated "mutant" and "wild-type" luciferase reporter constructs endowed either with four tandem copies of an 80-bp segment of the ovine GDF8 $3^{\prime}$ UTR centered around the

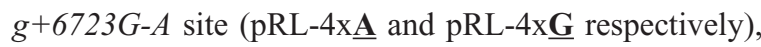
or with the complete ovine GDF8 $3^{\prime} \mathrm{UTR}$ (pRL-3' ${ }^{\prime} \mathbf{A}$ and pRL-3' $\underline{\mathbf{G}}$, respectively) in their $3^{\prime}$ UTR. Co-transfecting COS1 cells with these constructs and pcDNA3 vectors expressing either $m i R-1$ or $m i R-206$, or control miRNAs $m i R-136$ and $m i R-377$, demonstrated highly specific and significant reductions in luminescence signal when transfecting mutant $\underline{\mathbf{A}}$-constructs with $m i R-1$ and $m i R-206$ expressing vectors, but not when transfecting mutant $\underline{\mathbf{A}}-$ constructs with vectors expressing control miRNAs or when transfecting wild-type $\mathbf{G}$-constructs with either of the miRNA-expressing vectors.

Taken together, our results strongly support the fact that the $g+6723 G-A$ SNP is indeed the causative quanti- tative trait nucleotide (QTN) that creates a hypomorphic allele by promoting miRNA-mediated down-regulation of GDF8 and hence contributes to the muscular hypertrophy characteristic of Texel sheep (Fig.1).

\section{POLYMORPHIC MIRNA-TARGET INTERACTIONS: COMMON MEDIATORS OF PHENOTYPIC VARIATION?}

\section{Common Sequence Variants Alter the Content in Putative miRNA Target Site of Thousands of Human and Mouse Genes}

There is growing evidence that a substantial proportion of our genes (1/4th to $1 / 3 \mathrm{rd}$ ) are regulated by miRNAs (see, e.g., Lewis et al. 2005). Thus, the ovine $g+6723 G-A$ $G D F 8$ mutation-rather than being a sheep idiosyncrasy - may be the prototype example of a common type of polymorphisms that make a significant contribution to phenotypic variation. That this may indeed be the case is suggested by the recent description of a $G$ to $A$ substitution in the 3'UTR of the human SLITRK1 gene shown to be associated with Tourette's syndrome and thought to act by stabilizing the interaction with the coexpressed $m i R$ 189 miRNA (Abelson et al. 2005).

To evaluate how common polymorphic miRNA-target interactions might be, we examined whether known SNPs might alter the $3^{\prime}$ UTR content in miRNA target sites either by destroying existing sites or by creating new ones. The study was initially performed in human and mice, the two species for which the number of documented SNPs is largest. 92,967 human and 85,987 mouse SNPs, located, respectively, in 21,206 and 20,306 3'UTRs, were downloaded from Ensembl (http://www.ensembl.org/index.html). The ancestral allele was derived from the alignment with the chimpanzee for $95 \%$ of the human SNPs and from the alignment with the rat for $78 \%$ of the mouse SNPs.

miRNA target sites were defined on the basis of the collection of 540 octamers identified by Xie et al. (2005). Targets were labeled as evolutionarily conserved if the octamer was conserved across human, chimpanzee (only for mouse targets), mouse, rat (only for human targets), and dog; as nonconserved otherwise. The corresponding sequence alignments were downloaded from the UCSC genome browser (http://genome.ucsc.edu/).

We then searched for 3'UTR SNPs that would alter the target site content. Such SNPs will be hereafter referred to as pSNPs for Patrocles-SNPs. pSNPs for which the ancestral allele could be determined and that altered the target site content were classified in four groups: (1) destroying a conserved target site (DC), (2) destroying a nonconserved target site (DNC), (3) creating a nonconserved target site (CNC), or (4) shifting a target site (S). In some instances (1,235 in human; 4,278 in mouse), pSNPs were found that created a conserved site. We assumed that in these cases the allele shared by human and chimpanzee or mouse and rat was in fact a shared derived allele predating the corresponding species divergence. Hence, these pSNPs were reclassified as "DC." pSNPs changing target site content but for which the ancestral allele could not be 

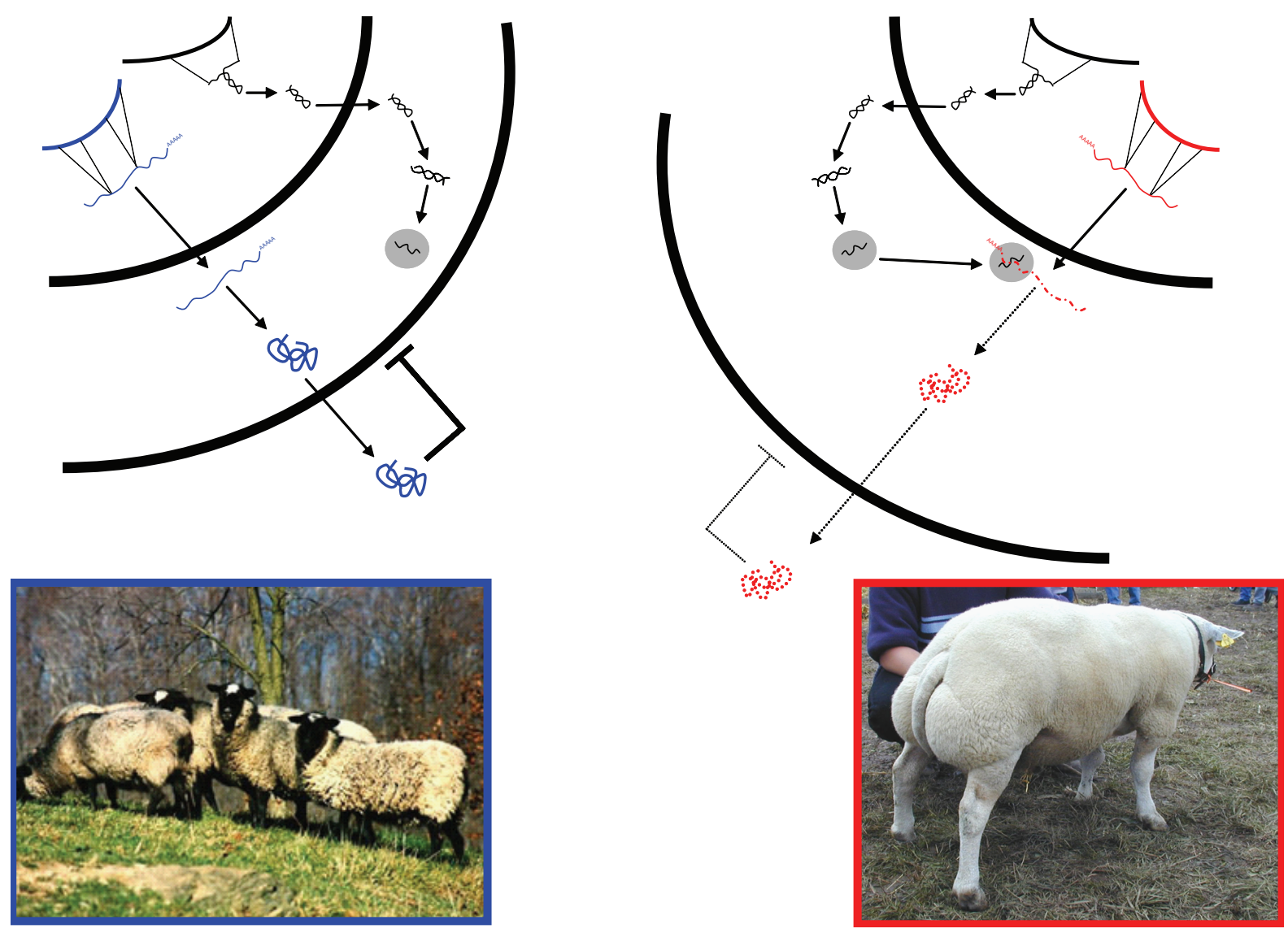

Figure 1. Schematic representation of the effect of the $g+6723 G-A$ mutation in the $3^{\prime} U T R$ of the ovine $G D F 8$ gene. In wild-type animals (such as the Romanov sheep shown in the left picture) the GDF8 transcripts (in blue) in the sarcoplasm do not interact with microRNAs $m i R-1$ and $m i R-206$ despite their high level of expression in skeletal muscle. Hence, GDF8 protein is produced at regular levels, controlling muscle growth by retro-inhibition. In mutant animals (such as the Texel sheep shown in the right picture), GDF8 transcripts (in red) become targets for $m i R-1$ and $m i R-206$ as a result of the $g+6723 G-A$ mutation. This leads to translational inhibition of the GDF8 transcripts, reduced GDF8 protein levels, relaxation of the retro-inhibition and, hence, enhanced muscle growth.

determined were classified as DC if one of the alleles was characterized by a conserved target site, or placed in a fifth category (polymorphic nonconserved- PNC) otherwise.

8,354 human pSNPs (i.e., 8.9\%) were found to affect the $3^{\prime}$ UTR target site content of 7,406 genes. The corresponding figures in the mouse were 7,942 SNPs in 4,582 genes. Table 1 reports the number and type of target site alterations caused by the corresponding sets of pSNPs. The total number of alterations exceeds the SNP numbers, as one SNP can have multiple effects.

Table 1. Numbers and Type of Target Site Alterations Caused by 8,354 human SNPs in the 3'UTRs of 7,406 Genes, and by 7,942 Mouse SNPs in the 3'UTR of 4,582 Genes

\begin{tabular}{|c|c|c|}
\hline & Conserved & Nonconserved \\
\hline Destroyed & $\begin{array}{l}\text { HS: } 500 \\
\text { MM: } 341\end{array}$ & $\begin{array}{r}\text { HS: } 4,014 \\
\text { MM: } 3,431\end{array}$ \\
\hline Created & $\begin{array}{l}\text { HS: } 0 \\
\text { MM: } 0\end{array}$ & $\begin{array}{r}\text { HS: } 4,544 \\
\text { MM: } 3,678\end{array}$ \\
\hline Polymorphic & & $\begin{array}{c}\text { HS: } 235 \\
\text { MM: } 1,327\end{array}$ \\
\hline Shifted & & \\
\hline
\end{tabular}

(HS) Human; (MM) mouse.

\section{Evidence for Purifying Selection against SNPs Altering the Target Site Content of $3^{\prime}$ UTRs}

Assuming that at least part of the predicted miRNA target sites are functional, pSNPs that alter these sites will likely affect gene function and may thus not be selectively neutral, being either subject to purifying selection if deleterious or to positive selection if beneficial. To provide evidence that at least some of the putative target sites- whether conserved or not-are selectively constrained, we compared the effects on target site content of the real set of human SNPs with the effects of simulated SNP sets. The simulated SNP sets were generated by randomly changing the position of each of the 92,967 human SNPs within the same gene. For example, a SNP corresponding to a $G$ to $A$ transition at position $\mathrm{x}$ of the $3^{\prime} \mathrm{UTR}$ of gene y would be randomly shifted toward any of the $G$ residues within the 3'UTR of gene y; a SNP corresponding to the insertion of a $\mathrm{G}$ residue would be randomly shifted toward any position of the corresponding 3'UTR; etc. One hundred such SNP sets were generated and subject to the same analysis as the set of real SNPs.

Figure 2 compares the distribution of the number of effects of different types obtained with the simulated and real data sets. The first noticeable observation is that the 
number of real pSNPs affecting target site content $(8,354)$ is 8.4 standard deviations below the mean number of pSNPs affecting target site content across simulated data sets $(9,155)$. This thus indicates that true SNPs are clearly "avoiding" putative miRNA target sites. This is either due to the fact that putative target sites are less prone to mutation, or more likely, that a significant proportion of putative target sites are under selective constraint. As expected, the category that is most markedly underrepresented in the real data set is the DC class: The true SNPs cause 500 such effects, which is 10 standard deviations below the average number of DC effects (783) observed with the simulated SNP sets. Conserved target sites have a higher likelihood of being genuine miRNA targets (or at least functional elements), and thus their destruction has a higher likelihood of being deleterious. More interesting is the lower than expected incidence of DNC cases with real SNPs $(4,014)$, 8.6 standard deviations below the equivalent number obtained on average $(4,595)$ across simulated SNP sets. This suggests that a substantial proportion of nonconserved target sites are selectively constrained and thus functional. This is somewhat unexpected, as the majority of nonconserved target sites are assumed to reside in genes that are not coexpressed with the cognate miRNA (see, e.g., Farh et al. 2005). Equally intriguing is the slightly lower than expected number of CNC cases (4,544 instead of 4,776, differing by 3.5 standard deviations), suggesting that a nonnegligible proportion of site creations is deleterious. Note that with the simulated SNP sets, the number of CNCs generally exceeds the number of DNCs by an average of 182 units. This could be due to the fact that the putative target sites are statistically underrepresented in the analyzed 3'UTR sequences, thus, that a random mutation has a higher likelihood to create than to destroy such a site. That this difference is more pronounced with the real SNPs (530 units) probably reflects stronger purifying selection against SNPs destroying than against SNPs creating nonconserved target sites. Finally, we observed twice as many $\mathrm{S}$ cases as expected ( 81 versus 38 , differing by 7.4 standard deviations). The reasons for this remain unclear.

\section{Patrocles: The Database of Polymorphic miRNA-Target Interactions}

To assist in the identification of SNPs that might affect gene function by perturbing miRNA-target interactions, we have developed a publicly accessible database that compiles information about polymorphisms that alter gene miRNA target site content in animals. The database is called Patrocles (http://www.patrocles.org), highlighting the parallel between the death of Patrocles and the down-regulation of $G D F 8$ in Texel as a result of the
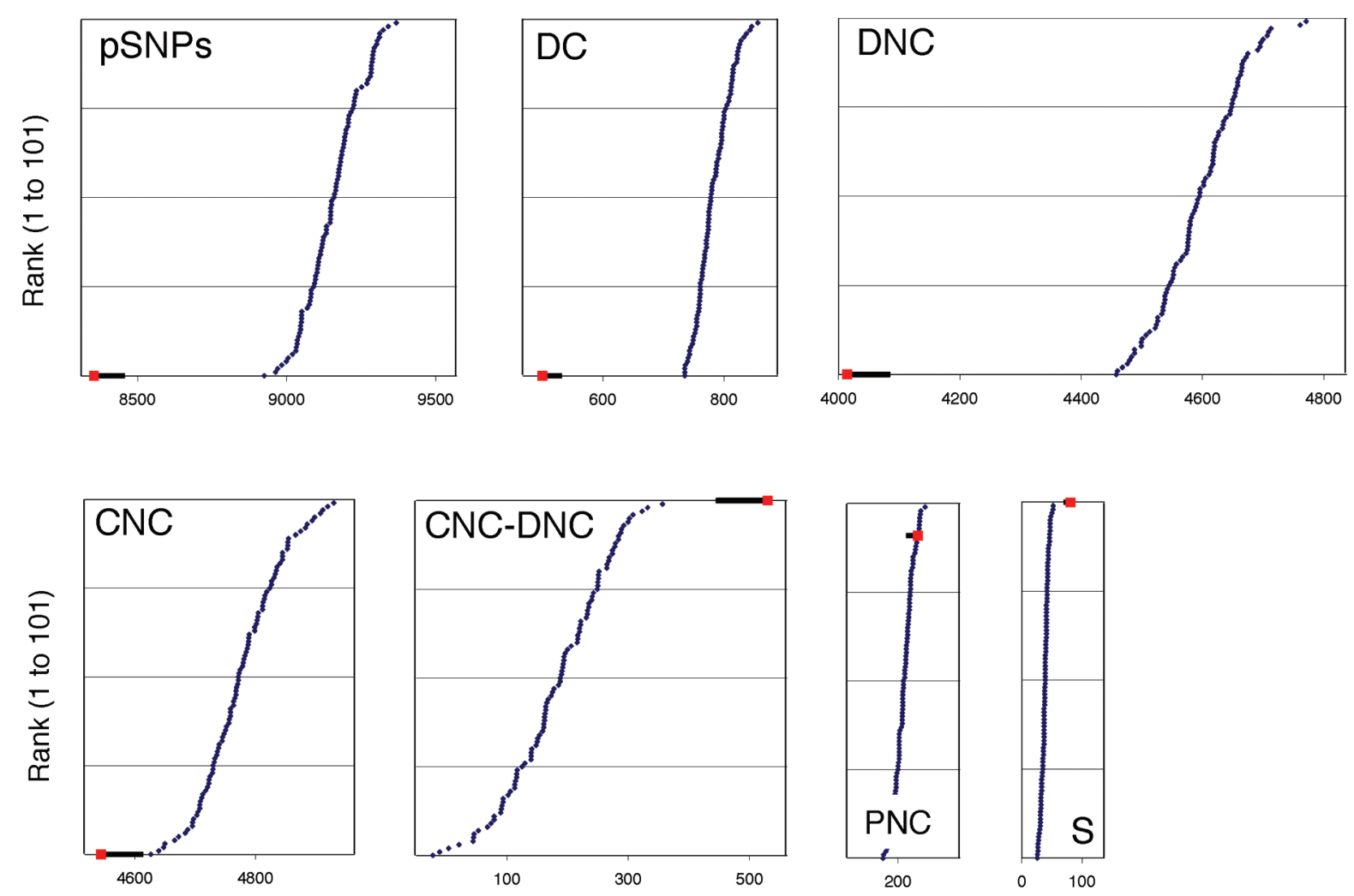

Figure 2. Distribution of the numbers of (1) SNPs altering miRNA target site content (pSNPs), (2) destructions of conserved target sites (DC), (3) destructions of nonconserved target sites (DNC), (4) creations of nonconserved target sites (CNC), (5) polymorphic target sites (PNC, ancestral allele unknown), and (6) shifted target sites (S). CNC-DNC corresponds to the difference in the number of creations versus destructions of nonconserved targets for a given SNP set. The red squares correspond to the numbers obtained with the real SNP set. The blue diamonds correspond to the numbers obtained with 100 simulated SNP sets. The black horizontal line corresponds to one standard deviation of the distribution across simulated data sets. Real and simulated data sets were ranked in ascending order. 
GEORGES ET AL.

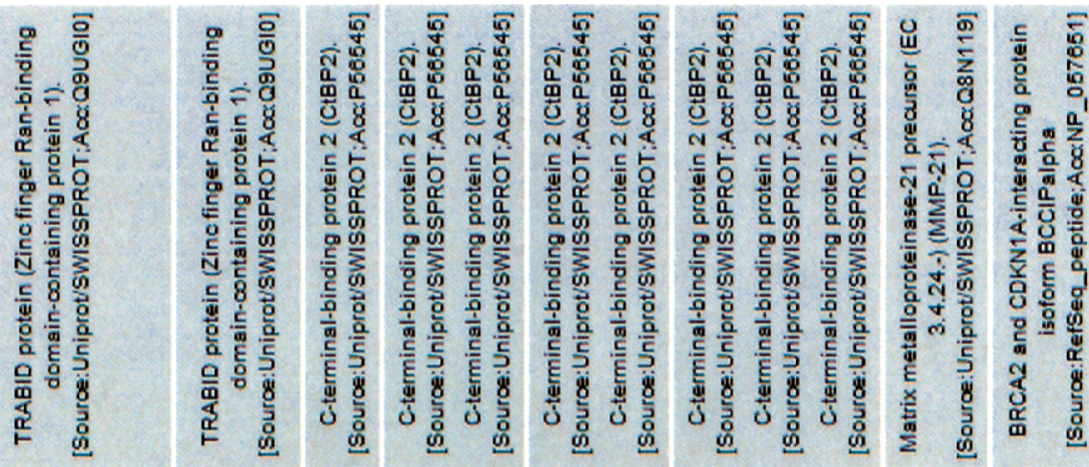

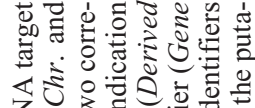

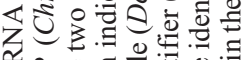

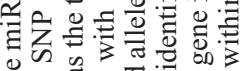

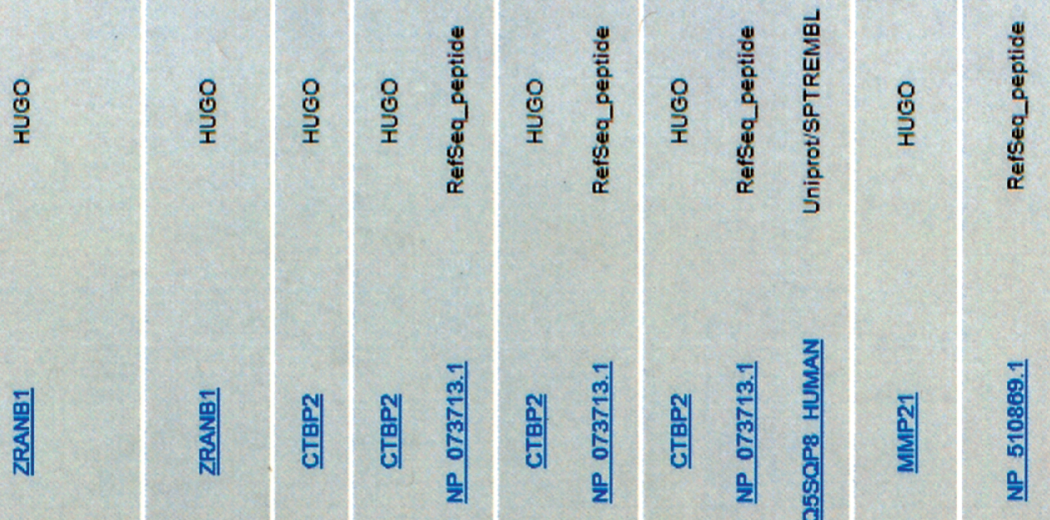

\begin{tabular}{|c|c|c|c|c|c|c|c|}
\hline 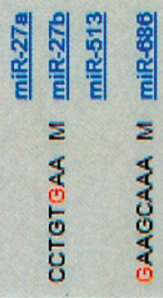 & & & & & 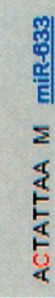 & & \\
\hline & 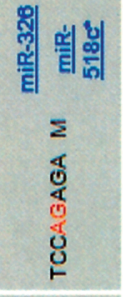 & 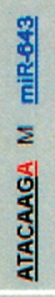 & 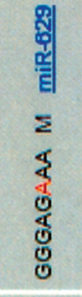 & 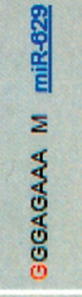 & & 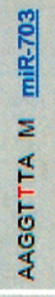 & 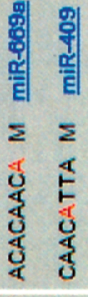 \\
\hline 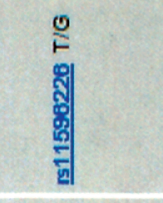 & $\begin{array}{l}\frac{1}{8} \\
\text { : } \\
\vdots \\
0 \\
0 \\
0\end{array}$ & 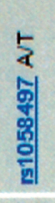 & 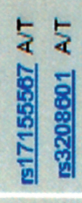 & 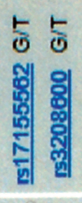 & 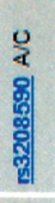 & 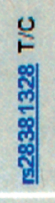 & 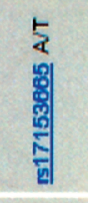 \\
\hline - & - & \ulcorner & 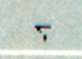 & $\div$ & 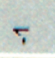 & 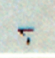 & - \\
\hline 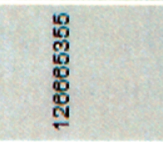 & 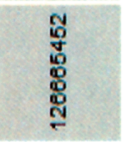 & 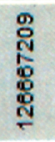 & $\begin{array}{l}8 \\
8 \\
0 \\
8 \\
8 \\
\text { ్․ }\end{array}$ & 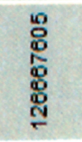 & 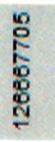 & $\begin{array}{l}\text { Nิ } \\
\text { ờ } \\
\text { స̃ } \\
\text { Na }\end{array}$ & 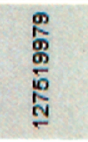 \\
\hline 우 & ? & ㅇ & 우 & ? & ? & ? & ? \\
\hline
\end{tabular}

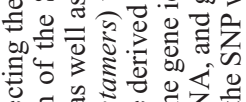
进.

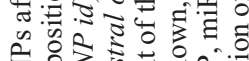

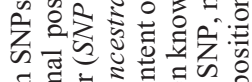
ज政击

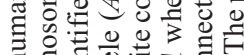

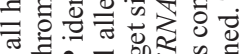

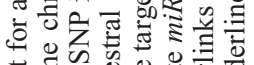
范的边。 행ㅎㅇㅇㅢ

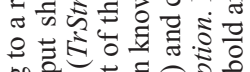

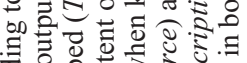

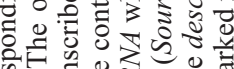
क्षे

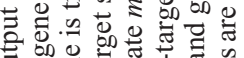

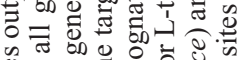

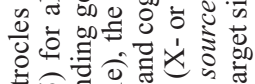

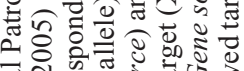

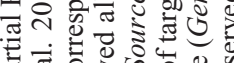
क न on. :

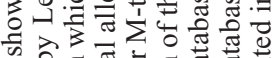

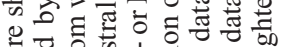

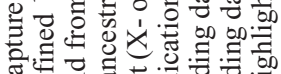

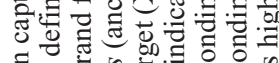

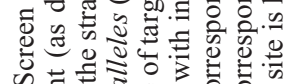
ผ

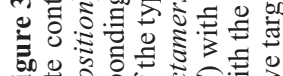

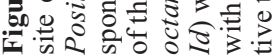


acquisition of Achilles' armor in the case of Patrocles and of an illegitimate miRNA target site in the case of GDF8.

In its present version, the database includes data about human, mouse, dog, chimpanzee, and bovine SNPs. As more SNP data become available for other species, these will be included in the database as well.

In addition to the effect of SNPs on the 3'UTR content in octamer motifs identified by Xie et al. (2005) (X-targets), Patrocles also compiles the effect of SNPs on miRNA target site content as defined by Lewis et al. (2005) (M-targets). These are composed of a heptamer exhibiting perfect Watson-Crick (WC) complementarity with bases 2-8 ("seeds") of known miRNAs, flanked by a t1 $A$ anchor on its $3^{\prime}$ side. This target site definition was selected to maximize specificity (Lewis et al. 2005). Known miRNAs were obtained from miRBase (http://microrna.sanger.ac.uk/sequences/index.shtml). The corresponding seeds were used across species, except for 47 miRNAs labeled as primate-specific which were only utilized to define human targets.

pSNPs destroying conserved target sites are likely to perturb gene function and hence to have phenotypic effects, including probably in some cases, to affect disease susceptibility. Equally interesting, however, are pSNPs that create target sites for miRNA that are expressed in the same tissue; i.e., SNPs creating a recognition site in an antitarget gene (Bartel and Chen 2004). The $g+6723 G-A$ mutation in the ovine $G D F 8$ gene and its effect on muscularity provide a good example of this type of pSNP. Moreover, if a pSNP destroys a nonconserved target site, it is certainly more likely to be biologically relevant if target gene and cognate miRNA have overlapping expression profiles. To facilitate the identification of truly interacting miRNA-gene pairs, we are in the process of adding information in Patrocles about the degree of coexpression of miRNA and genes. This is accomplished by confronting the experimentally determined gene expression profiles with computationally predicted miRNA expression profiles following Farh et al. (2005). The database can be queried by species, type of target site (X or L), effect of pSNP (DC, DNC, CNC, PNC, and S), pSNP identifier, gene identifier or chromosomal localization, miRNA identifier, or target site. The output provides information about the target content of the ancestral and derived alleles, including target site conservation and position of the pSNP in the target. Links are available for convenient mining of SNP and gene information. The output is available either on screen or can be downloaded as a text file (Fig. 3).

Further developments planned for the Patrocles database include the compilation of SNPs affecting the miRNA rather than the target, as well as the development of a tool to query the effect of a private SNP in a gene on putative miRNA-target interactions.

\section{CONCLUSIONS}

This work identifies a novel class of regulatory mutations that has the potential to make a significant contribution to the genetic variation for complex traits in many organisms, including disease susceptibility in man and agronomically important traits in animals and plants. The discovery of the $g+6723 G-A$ GDF8 mutation in sheep will encourage scientists to revisit their favorite positional and physiological candidate genes with a refreshed look in their quest for causative mutations. The evolving Patrocles database provides the community with a tool that should facilitate the identification of causative pSNPs.

\section{ACKNOWLEDGMENTS}

This project was supported by grants from (1) the Walloon Ministry of Agriculture (D31/1036), (2) the "GAME" Action de Recherche Concertée from the Communauté Française de Belgique, (3) the PAI P5/25 from the Belgian SSTC (R.SSTC.0135), (4) the European Union "Callimir" STREP project, and (5) the University of Liège. Alex Clop and Haruko Takeda both benefited from E.U. Marie-Curie postdoctoral fellowships. Carole Charlier is chercheur qualifié from the FNRS.

\section{REFERENCES}

Abelson J.F., Kwan K.Y., O’Roak B.J., Baek D.Y., Stillman A.A., Morgan T.M., Mathews C.A., Pauls D.L., Rasin M.R., Gunel M., et al. 2005. Sequence variants in SLITRK1 are associated with Tourette's syndrome. Science 310: 317.

Andersson L. and Georges M. 2004. Domestic animal genomics: Deciphering the genetics of complex traits. Nat. Rev. Genet. 5: 202.

Bartel D.P. and Chen C.Z. 2004. Micromanagers of gene expression: The potentially widespread influence of metazoan microRNAs. Nat. Rev. Genet. 5: 396.

Charlier C., Segers K., Karim L., Shay T., Gyapay G., Cockett N., and Georges M. 2001. The callipyge mutation enhances the expression of coregulated imprinted genes in cis without affecting their imprinting status. Nat. Genet. 27: 367.

Clop A., Marcq F., Takeda H., Pirottin D., Tordoir X., Bibe B., Bouix J., Caiment F., Elsen J.M., Eychenne F., et al. 2006. A mutation creating a potential illegitimate microRNA target site in the myostatin gene affects muscularity in sheep. Nat. Genet. 38: 813.

Davis E., Jensen C.H., Schroder H.D., Shay-Hsdfield T., Kliem A., Cockett N., Georges M., and Charlier C. 2004. Ectopic expression of DLK1 protein in skeletal muscle of padumnal heterozygotes causes the callipyge phenotype. Curr. Biol. 14: 1858 .

Doerge R.W. and Churchill G.A. 1996. Permutation tests for multiple loci affecting a quantitative character. Genetics 142: 285.

Farh K.K., Grimson A., Jan C., Lewis B.P., Johnston W.K., Lim L.P., Burge C.B., and Bartel D.P. 2005. The widespread impact of mammalian MicroRNAs on mRNA repression and evolution. Science 310: 1817.

Freking B.A., Murphy S.K., Wylie A.A., Rhodes S.J., Keele J.W., Leymaster K.A., Jirtle R.L., and Smith T.P. 2002. Identification of the single base change causing the callipyge muscle hypertrophy phenotype, the only known example of polar overdominance in mammals. Genome Res. 12: 1496.

Fujii J., Otsu K., Zorzato F., de Leon S., Khanna V.K., Weiler J.E., O'Brien P.J., and MacLennan D.H. 1991. Identification of a mutation in porcine ryanodine receptor associated with malignant hyperthermia. Science 253: 448.

Grobet L., Royo Martin L.J., Poncelet D., Pirottin D., Brouwers B., Riquet J., Schoeberlein A., Dunner S., Menissier F., Massabanda J., et al. 1997. A deletion in the myostatin gene causes double-muscling in cattle. Nat. Genet. 17: 71. 
Haley C.S., Knott S.A., and Elsen J.-M. 1994. Mapping quantitative trait loci in crosses between outbred lines using least squares. Genetics 136: 1195.

Lee S.J. 2004. Regulation of muscle mass by myostatin. Annu. Rev. Cell Dev. Biol. 20: 61

Lewis B.P., Burge C.B., and Bartel D.P. 2005. Conserved seed pairing, often flanked by adenosines, indicates that thousands of human genes are microRNA targets. Cell 120: 15 .

Lim L.P., Lau N.C., Garrett-Engele P., Grimson A., Schelter J.M., Castle J., Bartel D.P., Linsley P.S., and Johnson J.M. 2005. Microarray analysis shows that some microRNAs downregulate large numbers of target mRNAs. Nature 433: 769.

Schuelke M., Wagner K.R., Stolz L.E., Hubner C., Riebel T., Komen W., Braun T., Tobin J.F., and Lee S.J. 2004. Myostatin mutation associated with gross muscle hypertrophy in a child. N. Engl. J. Med. 350: 2682.
Van Laere A.-S., Nguyen M., Braunschweig M., Nezer C., Collette C., Moreau L., Archibald A.L., Haley C.S., Buys N., Tally M., et al. 2003. Positional identification of a regulatory mutation in $I G F 2$ causing a major QTL effect on muscle growth in the pig. Nature 425: 832 .

Visscher P.M., Thompson R., and Haley C.S. 1996. Confidence intervals in QTL mapping by bootstrapping. Genetics 143: 1013.

Xie X., Lu J., Kulbokas E.J., Golub T.R., Mootha V., LindbladToh K., Lander E.S., and Kellis M. 2005. Systematic discovery of regulatory motifs in human promoters and 3'UTRs by comparison of several mammals. Nature 434: 338.

Zimmers T.A., Davies M.V., Koniaris L.G., Haynes P., Esquela A.F., Tomkinson K.N., McPherron A.C., Wolfman N.M., and Lee S.J. 2002. Induction of cachexia in mice by systemically administered myostatin. Science 296: 1486. 


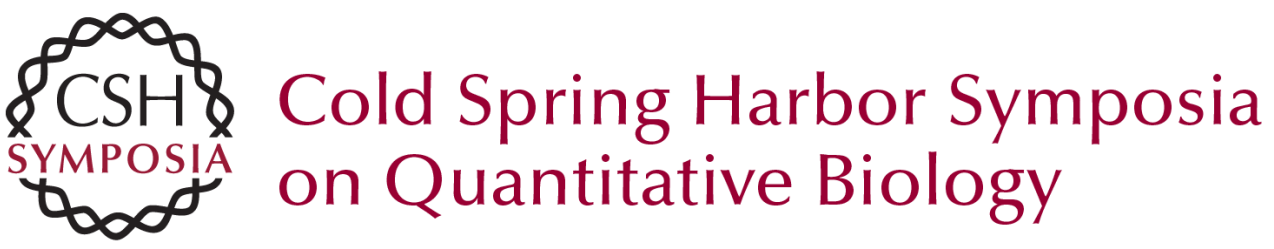

\section{Polymorphic Polymorphic MicroRNA-Target Interactions: A Novel Source of Phenotypic Variation}

M. GEORGES, A. CLOP, F. MARCQ, et al.

Cold Spring Harb Symp Quant Biol 2006 71: 343-350

Access the most recent version at doi:10.1101/sqb.2006.71.056

References This article cites 20 articles, 8 of which can be accessed free at:

http://symposium.cshlp.org/content/71/343.full.html\#ref-list-1

License

Email Alerting Receive free email alerts when new articles cite this article - sign up in

Service the box at the top right corner of the article or click here. 